\title{
PVT Investigation of an Iraqi Reservoir
}

\author{
Jihad Husain Al-Joumaa*; Dr.Mohammed S. Al-Jawad*; Craig W. Van Kirk** \\ * Department of Petroleum Engineering/UoT \\ **Colorado School of Mines.
}

\section{$\underline{\text { Abstract }}$}

This investigation was made to clarify the type of reservoir fluids in an Iraqi field reservoir regarding the controversy about its type. It was known for many years as a condensate field, but in this study a different theory was investigated and proved using Winprop of $\mathrm{CMG}^{\circledR}$ software.

Two equations of state (EOS) were tested and the one with minimum error percentage was used. Also, three correlations were tested to calculate the original $\mathrm{C}_{6}{ }^{+}$properties, which are: LeeKesler, Twu and Riazi. Twu`s correlation prevailed because the results of this correlation were the nearest to the original data, and it permits using a wide range of pressures (from zero to infinite pressure values), which minimizes the discrepancies between the results of any equation of state, which was investigated.

After achieving the closest possible match of all available properties, the envelopes were constructed. Winprop of CMG needs a full set of oil and gas properties from a single sample. Therefore, a recombination for the available gas and oil samples were made to construct the phase envelopes for the field system in addition to individual gas and oil phase envelopes

The result of this investigation and analysis is that the reservoir is actually a black oil field with a gas cap, despite what was believed earlier as being a condensate field.

Keywords: EOS, Correlation, Plus fraction properties $\left(\mathrm{C}_{6}{ }^{+}\right)$, Regression, Phase Envelopes, Black Oil (not condensate) reservoir. 


\section{No.16 Journal of Petroleum Research \& Studies}

\section{(JPR\&S)}

\section{Introduction}

PVT analysis (which means pressure, volume, and temperature) is an examination of hydrocarbon reservoir fluids in a laboratory under various pressures, volumes, temperatures and compositions to determine the physical characteristics and behavior of the fluid [1].

The PVT analyses are first steps in any reservoir study and are important to determine the type of the fluids concerned with the study, and to predict their physical properties in order to select an appropriate simulator to build the reservoir model of that field for history matching and/or forecasting.

Winprop of CMG software is one of the most up-to-date programs in analyzing PVT data. It uses two equations of state and correlations for original properties and $\mathrm{C}+$ properties.

The two equations of states that were tested are: Peng-Robinson (PR) [2] and Soave Redlich Kwang (SRK) [3]. Choosing the equation of state and correlations depends on the minimum error percentage result in each step.

The EOS needs very accurate properties of $\mathrm{C}_{6}{ }^{+}$in order to give correct results, and because there are no $\mathrm{C}_{6}{ }^{+}$data, a tuning process must be made.

A regression method [4] which is an option in the Winprop program was used to calculate the properties of the $\mathrm{C}_{6}^{+}$analysis and many factors.

The tuning process or "Regression analysis" is useful when the model parameters arising from the basic characterization procedure do not result in the desired agreement with PVT data, which is basically because there are no specific properties for the plus hydrocarbon fractions $\left(\mathrm{C}_{6}{ }^{+}\right)$, so the regression minimizes the deviation between the measured data and the simulated results of the PVT experiments by varying a selection of model parameters.

It is important to note that the three correlations were tested to calculate the original $\mathrm{C}_{6}{ }^{+}$ properties, which are: Lee-Kesler [5] , Twu [6] and Riazi [7] 


\section{No.16 Journal of Petroleum Research \& Studies}

\section{(JPR\&S)}

Using all the previous steps and information, a phase envelop can be constructed. Based on the resulting phase envelopes, the types of the fluids in the field can be identified. Oil, gas, and recombination phases can be constructed based on the available data and the particular needs of each reservoir study [8].

\section{Phase Behavior Investigation}

The available PVT data of the reservoir for wells X-5, X-8, X-2, X-43, X-47, X-52 and X-26 are: Bo (oil formation-volume factor), $\mathrm{Bg}$ (gas formation-volume factor), GOR (solution gas-oil ratio) and ROV (relative oil volume) vs. P (pressure) are shown in Figures (1-4), and the values of hydrocarbon weight percent as listed in Tables (1\&2). Also, values of bubble-point pressure and the reservoir temperature must be prepared in order to export them to Winprop of CMG.

Other available information used for both oil and gas wells were as follows; for oil wells: the saturation pressure is $3750 \mathrm{psia}$ and the reservoir temperature is $146{ }^{\circ} \mathrm{F}$, the sampling conditions are 3600 psia pressure and $146{ }^{\circ} \mathrm{F}$ temperature, the production conditions (separators) are 70 psia for pressure and $70{ }^{\circ} \mathrm{F}$ for temperature.

The reservoir conditions for gas wells are 3750 psia saturation pressure and $145^{\circ} \mathrm{F}$ reservoir temperature, the sampling conditions are 3680 psia pressure and $145{ }^{\circ} \mathrm{F}$ temperature, the production conditions (separators) are 80 psia pressure and $80{ }^{\circ} \mathrm{F}$ temperature.

The SRK EOS has less error percentage in matching the real data of the field, because the PR EOS fails in determining the correct Bp pressure for the reservoir. Using Twu's correlation to calculate the $\mathrm{C}_{6}{ }^{+}$properties also gives the nearest values to the original properties. Evaluation of the final properties is the regression method as shown in Tables (3\&4).

Eight phase envelopes for the reservoir were obtained using the recombination of available PVT reports of oil and gas, and a ninth phase envelop was determined from the composition of reservoir crude of the PVT report for well X-26.

All nine phase envelopes were identical, and the result was that the reservoir is not a condensate fluid, but it is a saturated black-oil field with a gas cap having high percentage of $\mathrm{C}_{1}$. 
Figure (1) to Figure (4) is comparisons between PR and SRK matching regarding GOR, ROV, Oil and Gas viscosity. Figure (5) through Figure (7) represents the phase envelopes for the reservoir, oil and gas respectively.

Gas wells phase envelopes indicate that the gas is dry with a high percentage of $\mathrm{C}_{1}$, and the oil phase envelope indicates that the fluid is black oil.

The matching between the real and resulting data was very similar to the recombination results with a highest error percentage of $3.7 \%$ for all the relations according to the SRK method, as shown in Table (5).

Table (1) Hydrocarbon weight percentage for gas wells.

\begin{tabular}{|c|c|c|c|}
\hline & X-5 & X-8 & X-26 \\
\hline Composition & Weight \% & Weight \% & Weight \% \\
\hline C1 & 75.08 & 79.4 & 75.000 \\
\hline C2 & 9.52 & 1.4 & 4.4027 \\
\hline C3 & 5.31 & 1.2 & 0.7562 \\
\hline iC4 & 3.62 & 3.6 & 2.320 \\
\hline nC4 & 1.07 & 5.3 & 4.8977 \\
\hline iC5 & 1.83 & 3.4 & 2.2399 \\
\hline nC5 & 1.65 & 3.9 & 3.5197 \\
\hline C6+ & 1.92 & 1.8 & 6.8653 \\
\hline Sum & 100.00 & 100.0 & 100.000 \\
\hline
\end{tabular}




\section{No.16 Journal of Petroleum Research \& Studies}

\section{(JPR\&S)}

Table (2) Hydrocarbon weight percentage for oil wells.

\begin{tabular}{|c|c|c|c|c|c|}
\hline & X-2 & X-43 & X-47 & X-52 & X-26 \\
\hline Composition & Weight \% & Weight $\%$ & Weight \% & Weight \% & Weight \% \\
\hline C1 & 0.53 & 0.30 & 0.56 & 0.7 & 1.22 \\
\hline C2 & 0.69 & 0.32 & 0.66 & 0.9 & 3.38 \\
\hline C3 & 0.77 & 0.51 & 0.73 & 1.1 & 3.72 \\
\hline iC4 & 1.23 & 0.97 & 1.27 & 1.8 & 1.87 \\
\hline nC4 & 1.99 & 4.86 & 1.91 & 1.9 & 2.46 \\
\hline iC5 & 5.47 & 7.75 & 5.79 & 5.5 & 1.87 \\
\hline $\mathrm{nC5}$ & 7.37 & 9.40 & 7.36 & 10.8 & 9.73 \\
\hline C6+ & 81.95 & 75.89 & 81.72 & 77.3 & 75.75 \\
\hline Sum & 100.00 & 100.00 & 100.00 & 100.0 & 100.00 \\
\hline
\end{tabular}

Table (3) SRK parameters.

\begin{tabular}{|c|c|c|c|c|c|c|c|c|c|}
\hline Component & $\begin{array}{c}\text { Pc } \\
\text { (atm) }\end{array}$ & $\begin{array}{c}\text { Tc } \\
\text { (k) }\end{array}$ & $\begin{array}{c}\text { Acentric } \\
\text { factor }\end{array}$ & $\begin{array}{c}\text { Mole } \\
\text { weight }\end{array}$ & $\begin{array}{c}\text { volume } \\
\text { shift }\end{array}$ & $\begin{array}{c}\mathbf{Z} \\
\text { (rackett) }\end{array}$ & $\begin{array}{c}\text { Vc } \\
\text { (l/mol) }\end{array}$ & viscosity & Sg \\
\hline C1 & 45.4 & 190.6 & 0.008 & 16.043 & 0 & 0.2876 & 0.099 & 0.099 & 0.300 \\
\hline C2 & 48.2 & 305.4 & 0.098 & 30.07 & 0 & 0.2789 & 0.148 & 0.148 & 0.356 \\
\hline C3 & 41.9 & 369.8 & 0.152 & 44.097 & 0 & 0.2763 & 0.203 & 0.203 & 0.507 \\
\hline IC4 & 36.0 & 408.1 & 0.176 & 58.124 & 0 & 0.2750 & 0.263 & 0.263 & 0.563 \\
\hline NC4 & 37.5 & 425.2 & 0.193 & 58.124 & 0 & 0.2728 & 0.255 & 0.255 & 0.584 \\
\hline IC5 & 33.4 & 460.4 & 0.227 & 72.151 & 0 & 0.2716 & 0.306 & 0.306 & 0.625 \\
\hline NC5 & 33.3 & 469.6 & 0.251 & 72.151 & 0 & 0.2685 & 0.304 & 0.304 & 0.631 \\
\hline $\begin{array}{c}\text { C6+ } \\
\text { (original) }\end{array}$ & 32.86 & 489.5474 & 0.330048 & 85.99991 & 0.220455 & 0.27648 & 0.4128 & 0.344 & 0.69 \\
\hline $\begin{array}{c}\text { C6+ } \\
\text { (regression) }\end{array}$ & 30.9463 & 480.0986 & 0.396058 & 85.99991 & 0.237644 & 0.268979 & 0.4128 & 0.344 & 0.69 \\
\hline
\end{tabular}




\section{No.16 Journal of Petroleum Research \& Studies}

\section{(JPR\&S)}

\begin{tabular}{|c|c|c|c|c|c|c|c|c|}
\hline Component & $\begin{array}{c}\text { Omega } \\
\text { A }\end{array}$ & Omega B & $\begin{array}{c}\text { Enth. } \\
\text { Coef. A }\end{array}$ & $\begin{array}{c}\text { Enth. } \\
\text { Coef. B }\end{array}$ & $\begin{array}{c}\text { Enth. } \\
\text { Coef. C }\end{array}$ & $\begin{array}{c}\text { Enth. } \\
\text { Coef. D }\end{array}$ & $\begin{array}{c}\text { Enth. } \\
\text { Coef. E }\end{array}$ & $\begin{array}{c}\text { Enth. } \\
\text { Coef. F }\end{array}$ \\
\hline $\mathbf{C 1}$ & 0.42748 & 0.08664 & $0.00 \mathrm{E}+00$ & $1.96 \mathrm{E}-01$ & $3.89 \mathrm{E}-04$ & $-5.13 \mathrm{E}-08$ & $0.00 \mathrm{E}+00$ & $0.00 \mathrm{E}+00$ \\
\hline $\mathbf{C} 2$ & 0.42748 & 0.08664 & $0.00 \mathrm{E}+00$ & $1.95 \mathrm{E}-01$ & $3.90 \mathrm{E}-04$ & $-5.13 \mathrm{E}-08$ & $0.00 \mathrm{E}+00$ & $0.00 \mathrm{E}+00$ \\
\hline $\mathbf{C 3}$ & 0.42748 & 0.08664 & $0.00 \mathrm{E}+00$ & $6.78 \mathrm{E}-02$ & $4.11 \mathrm{E}-04$ & $-5.13 \mathrm{E}-08$ & $0.00 \mathrm{E}+00$ & $0.00 \mathrm{E}+00$ \\
\hline $\mathbf{I C 4}$ & 0.42748 & 0.08664 & $0.00 \mathrm{E}+00$ & $4.35 \mathrm{E}-02$ & $4.05 \mathrm{E}-04$ & $-5.13 \mathrm{E}-08$ & $0.00 \mathrm{E}+00$ & $0.00 \mathrm{E}+00$ \\
\hline $\mathbf{N C 4}$ & 0.42748 & 0.08664 & $0.00 \mathrm{E}+00$ & $3.52 \mathrm{E}-02$ & $4.02 \mathrm{E}-04$ & $-5.13 \mathrm{E}-08$ & $0.00 \mathrm{E}+00$ & $0.00 \mathrm{E}+00$ \\
\hline $\mathbf{I C 5}$ & 0.42748 & 0.08664 & $0.00 \mathrm{E}+00$ & $2.28 \mathrm{E}-02$ & $3.98 \mathrm{E}-04$ & $-5.13 \mathrm{E}-08$ & $0.00 \mathrm{E}+00$ & $0.00 \mathrm{E}+00$ \\
\hline $\mathbf{N C 5}$ & 0.42748 & 0.08664 & $0.00 \mathrm{E}+00$ & $2.26 \mathrm{E}-02$ & $3.98 \mathrm{E}-04$ & $-5.13 \mathrm{E}-08$ & $0.00 \mathrm{E}+00$ & $0.00 \mathrm{E}+00$ \\
\hline $\begin{array}{c}\mathbf{C 6}+ \\
\text { (original) }\end{array}$ & 0.42700 & 0.08664 & $0.00 \mathrm{E}+00$ & $-1.63 \mathrm{E}-02$ & $4.12 \mathrm{E}-04$ & $-5.77 \mathrm{E}-08$ & $0.00 \mathrm{E}+00$ & $0.00 \mathrm{E}+00$ \\
\hline $\begin{array}{c}\mathbf{C} 6+ \\
\text { (regression) }\end{array}$ & 0.60098 & 0.077182 & $0.00 \mathrm{E}+00$ & $-1.63 \mathrm{E}-02$ & $4.12 \mathrm{E}-04$ & $-5.77 \mathrm{E}-08$ & $0.00 \mathrm{E}+00$ & $0.00 \mathrm{E}+00$ \\
\hline
\end{tabular}

Table (4) PR parameters

\begin{tabular}{|c|c|c|c|c|c|c|c|c|c|}
\hline Component & $\begin{array}{c}\text { Pc } \\
\text { (atm) }\end{array}$ & $\begin{array}{c}\text { Tc } \\
\text { (k) }\end{array}$ & $\begin{array}{c}\text { Acentric } \\
\text { factor }\end{array}$ & $\begin{array}{c}\text { Mole } \\
\text { weight }\end{array}$ & $\begin{array}{c}\text { volume } \\
\text { shift }\end{array}$ & $\begin{array}{c}\mathbf{Z} \\
\text { (rackett) }\end{array}$ & $\begin{array}{c}\text { Vc } \\
\text { (l/mol) }\end{array}$ & (viscosity) & Sg \\
\hline C1 & 45.4 & 190.6 & 0.008 & 16.043 & 0 & 0.2876 & 0.099 & 0.099 & 0.300 \\
\hline C2 & 48.2 & 305.4 & 0.098 & 30.07 & 0 & 0.2789 & 0.148 & 0.148 & 0.356 \\
\hline C3 & 41.9 & 369.8 & 0.152 & 44.097 & 0 & 0.2763 & 0.203 & 0.203 & 0.507 \\
\hline IC4 & 36.0 & 408.1 & 0.176 & 58.124 & 0 & 0.2750 & 0.263 & 0.263 & 0.563 \\
\hline NC4 & 37.5 & 425.2 & 0.193 & 58.124 & 0 & 0.2728 & 0.255 & 0.255 & 0.584 \\
\hline IC5 & 33.4 & 460.4 & 0.227 & 72.151 & 0 & 0.2716 & 0.306 & 0.306 & 0.625 \\
\hline NC5 & 33.3 & 469.6 & 0.251 & 72.151 & 0 & 0.2685 & 0.304 & 0.304 & 0.631 \\
\hline $\begin{array}{c}\text { C6+ } \\
\text { (original) }\end{array}$ & 32.86 & 489.5474 & 8 & 85.99991 & 0.220455 & 0.27648 & 0.4128 & 0.344 & 0.69 \\
\hline $\begin{array}{c}\text { C6+ } \\
\text { (regression) }\end{array}$ & 32.86 & 533.7999 & 8 & 85.99991 & 0.215828 & 0.268317 & 0.4128 & 0.344 & 0.69 \\
\hline
\end{tabular}




\section{No.16 Journal of Petroleum Research \& Studies}

\section{(JPR\&S)}

\begin{tabular}{|c|c|c|c|c|c|c|c|c|}
\hline Component & $\begin{array}{c}\text { Omega } \\
\text { A }\end{array}$ & Omega B & $\begin{array}{c}\text { Enth. } \\
\text { Coef. A }\end{array}$ & $\begin{array}{c}\text { Enth. } \\
\text { Coef. B }\end{array}$ & $\begin{array}{c}\text { Enth. } \\
\text { Coef. C }\end{array}$ & $\begin{array}{c}\text { Enth. } \\
\text { Coef. D }\end{array}$ & $\begin{array}{c}\text { Enth. } \\
\text { Coef. E }\end{array}$ & $\begin{array}{l}\text { Enth. } \\
\text { Coef. F }\end{array}$ \\
\hline C1 & $\begin{array}{c}4.57 \mathrm{E}- \\
01\end{array}$ & $7.78 \mathrm{E}-02$ & $0.00 \mathrm{E}+00$ & $1.96 \mathrm{E}-01$ & $3.89 \mathrm{E}-04$ & $-5.13 \mathrm{E}-08$ & $0.00 \mathrm{E}+00$ & $0.00 \mathrm{E}+00$ \\
\hline $\mathrm{C} 2$ & $\begin{array}{c}4.57 \mathrm{E}- \\
01\end{array}$ & $7.78 \mathrm{E}-02$ & $0.00 \mathrm{E}+00$ & $1.95 \mathrm{E}-01$ & $3.90 \mathrm{E}-04$ & $-5.13 \mathrm{E}-08$ & $0.00 \mathrm{E}+00$ & $0.00 \mathrm{E}+00$ \\
\hline C3 & $\begin{array}{c}4.57 \mathrm{E}- \\
01 \\
\end{array}$ & $7.78 \mathrm{E}-02$ & $0.00 \mathrm{E}+00$ & $6.78 \mathrm{E}-02$ & $4.11 \mathrm{E}-04$ & $-5.13 \mathrm{E}-08$ & $0.00 \mathrm{E}+00$ & $0.00 \mathrm{E}+00$ \\
\hline IC4 & $\begin{array}{c}4.57 \mathrm{E}- \\
01 \\
\end{array}$ & $7.78 \mathrm{E}-02$ & $0.00 \mathrm{E}+00$ & 4.35E-02 & $4.05 \mathrm{E}-04$ & $-5.13 \mathrm{E}-08$ & $0.00 \mathrm{E}+00$ & $0.00 \mathrm{E}+00$ \\
\hline $\mathrm{NC4}$ & $\begin{array}{c}4.57 \mathrm{E}- \\
01\end{array}$ & $7.78 \mathrm{E}-02$ & $0.00 \mathrm{E}+00$ & $3.52 \mathrm{E}-02$ & 4.02E-04 & $-5.13 \mathrm{E}-08$ & $0.00 \mathrm{E}+00$ & $0.00 \mathrm{E}+00$ \\
\hline IC5 & $\begin{array}{c}4.57 \mathrm{E}- \\
01\end{array}$ & $7.78 \mathrm{E}-02$ & $0.00 \mathrm{E}+00$ & $2.28 \mathrm{E}-02$ & $3.98 \mathrm{E}-04$ & $-5.13 \mathrm{E}-08$ & $0.00 \mathrm{E}+00$ & $0.00 \mathrm{E}+00$ \\
\hline NC5 & $\begin{array}{c}4.57 \mathrm{E}- \\
01\end{array}$ & $7.78 \mathrm{E}-02$ & $0.00 \mathrm{E}+00$ & $2.26 \mathrm{E}-02$ & $3.98 \mathrm{E}-04$ & $-5.13 \mathrm{E}-08$ & $0.00 \mathrm{E}+00$ & $0.00 \mathrm{E}+00$ \\
\hline $\begin{array}{c}\mathrm{C6}^{+} \\
\text {(original) }\end{array}$ & $\begin{array}{c}4.57 \mathrm{E}- \\
01 \\
\end{array}$ & $7.78 \mathrm{E}-02$ & $0.00 \mathrm{E}+00$ & $\begin{array}{c}-1.63 \mathrm{E}- \\
02 \\
\end{array}$ & $4.12 \mathrm{E}-04$ & $-5.77 \mathrm{E}-08$ & $0.00 \mathrm{E}+00$ & $0.00 \mathrm{E}+00$ \\
\hline $\begin{array}{c}\text { C6+ } \\
\text { (regression) }\end{array}$ & 0.60098 & $7.78 \mathrm{E}-02$ & $0.00 \mathrm{E}+00$ & $\begin{array}{c}-1.63 \mathrm{E}- \\
02\end{array}$ & 4.12E-04 & $-5.77 \mathrm{E}-08$ & $0.00 \mathrm{E}+00$ & $0.00 \mathrm{E}+00$ \\
\hline
\end{tabular}

Table (5) Error percentage in fluid properties between PR and SRK EOS

\begin{tabular}{|c|c|c|c|c|c|}
\hline & GOR & ROV & Gas FVF & Oil Viscosity & Gas Viscosity \\
\hline PR (avg.) & $7 \%$ & $6 \%$ & $5 \%$ & $6 \%$ & $4 \%$ \\
\hline PR (highest) & $11 \%$ & $9 \%$ & $6 \%$ & $10 \%$ & $10 \%$ \\
\hline SRK (avg.) & $2 \%$ & $1 \%$ & $3 \%$ & $2 \%$ & $1 \%$ \\
\hline SRK (highest) & $4 \%$ & $2 \%$ & $4 \%$ & $4 \%$ & $3 \%$ \\
\hline
\end{tabular}




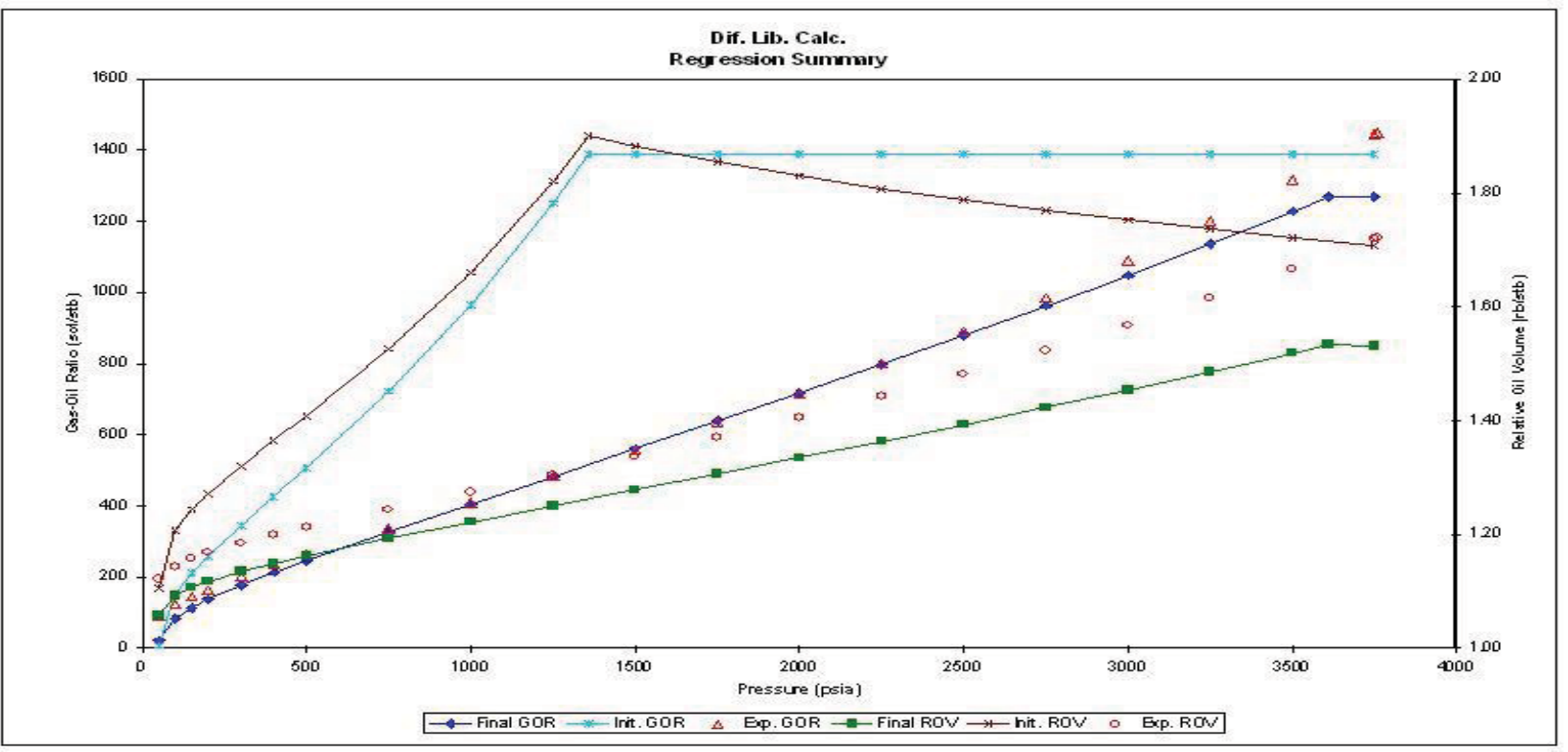

Fig.(1) GOR, ROV vs. P (PR method)

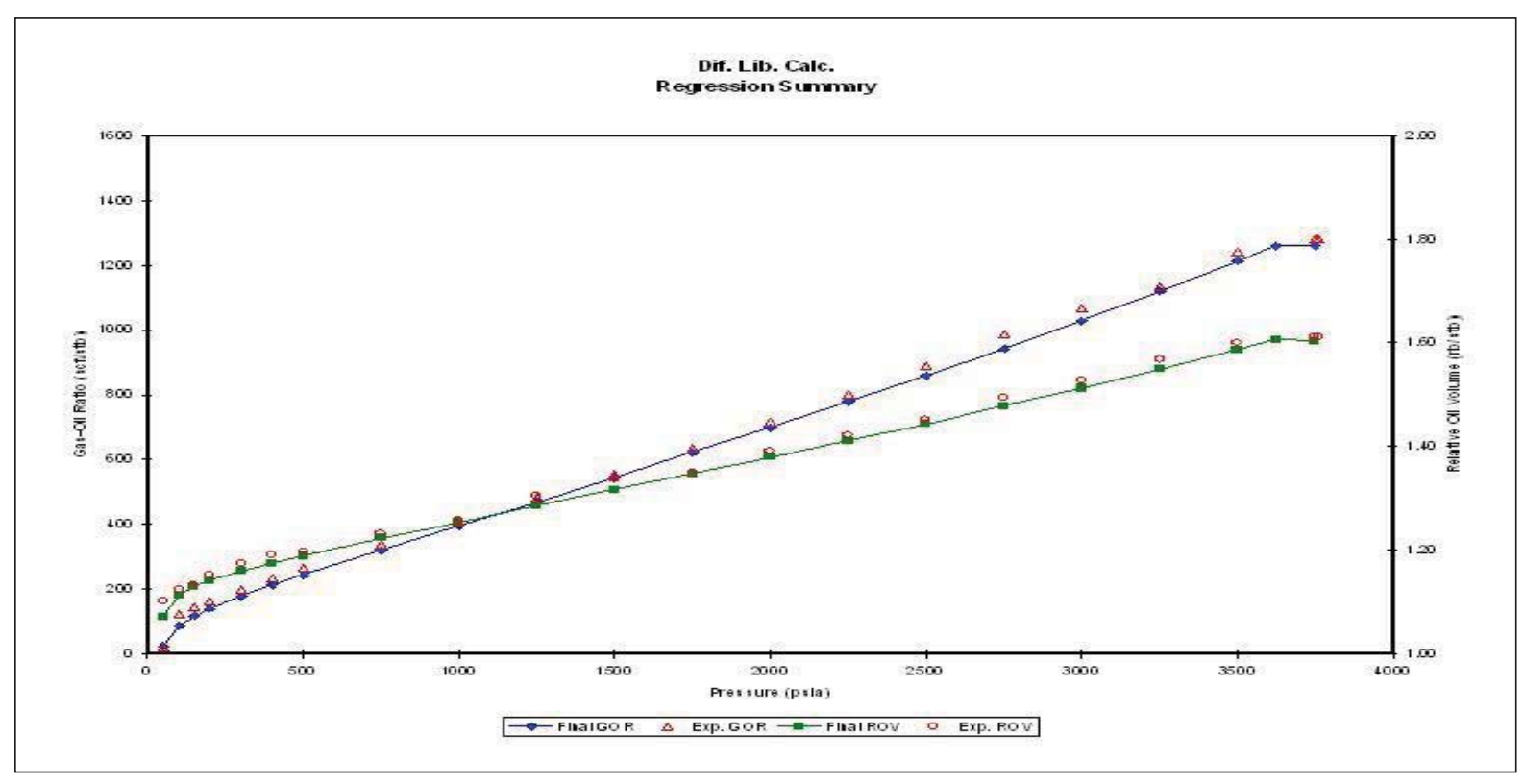

Fig.(2) GOR, ROV vs. P (SRK method) 


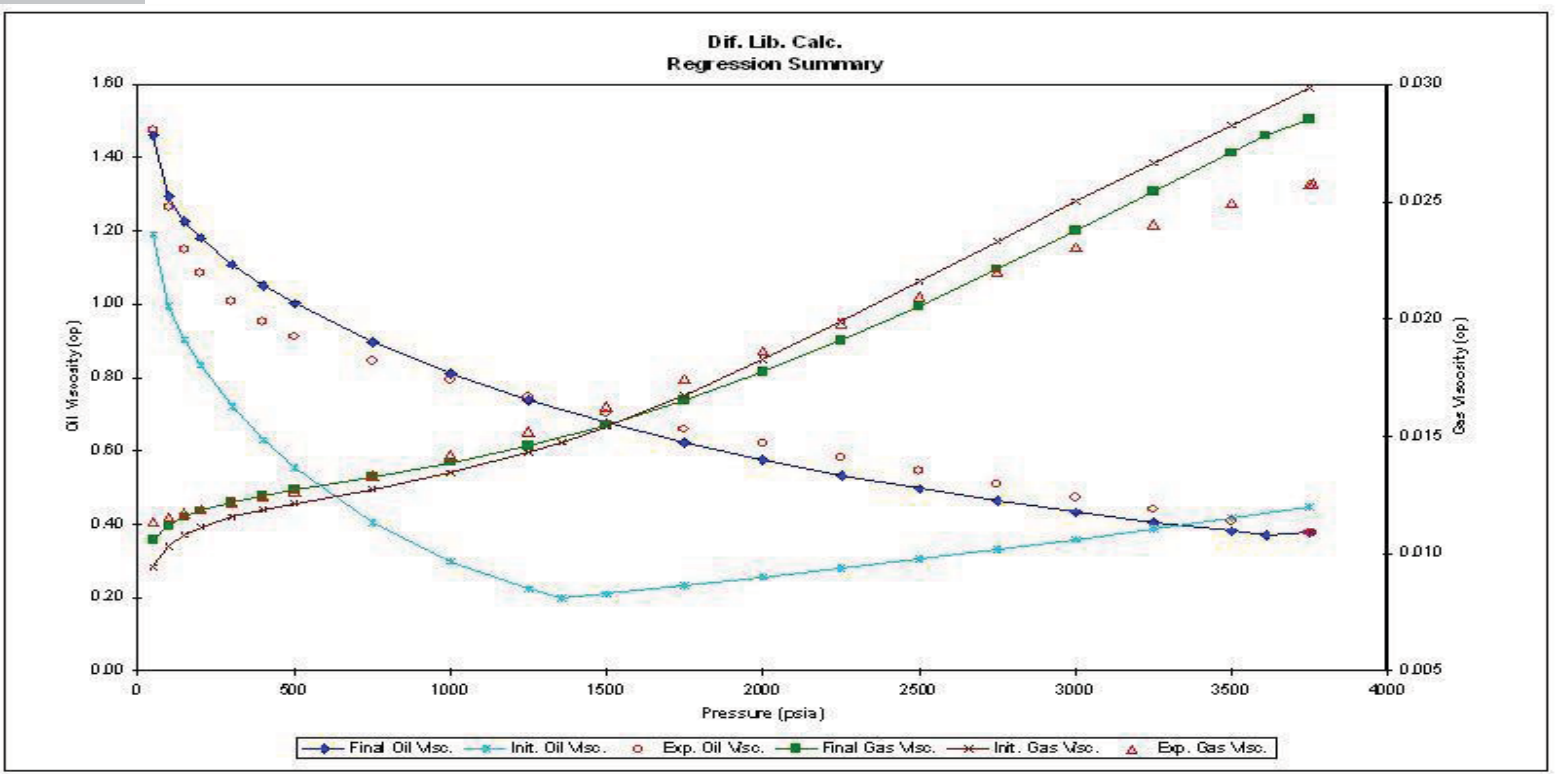

Fig. (3) Oil Viscosity, Gas Viscosity vs. P (PR method)

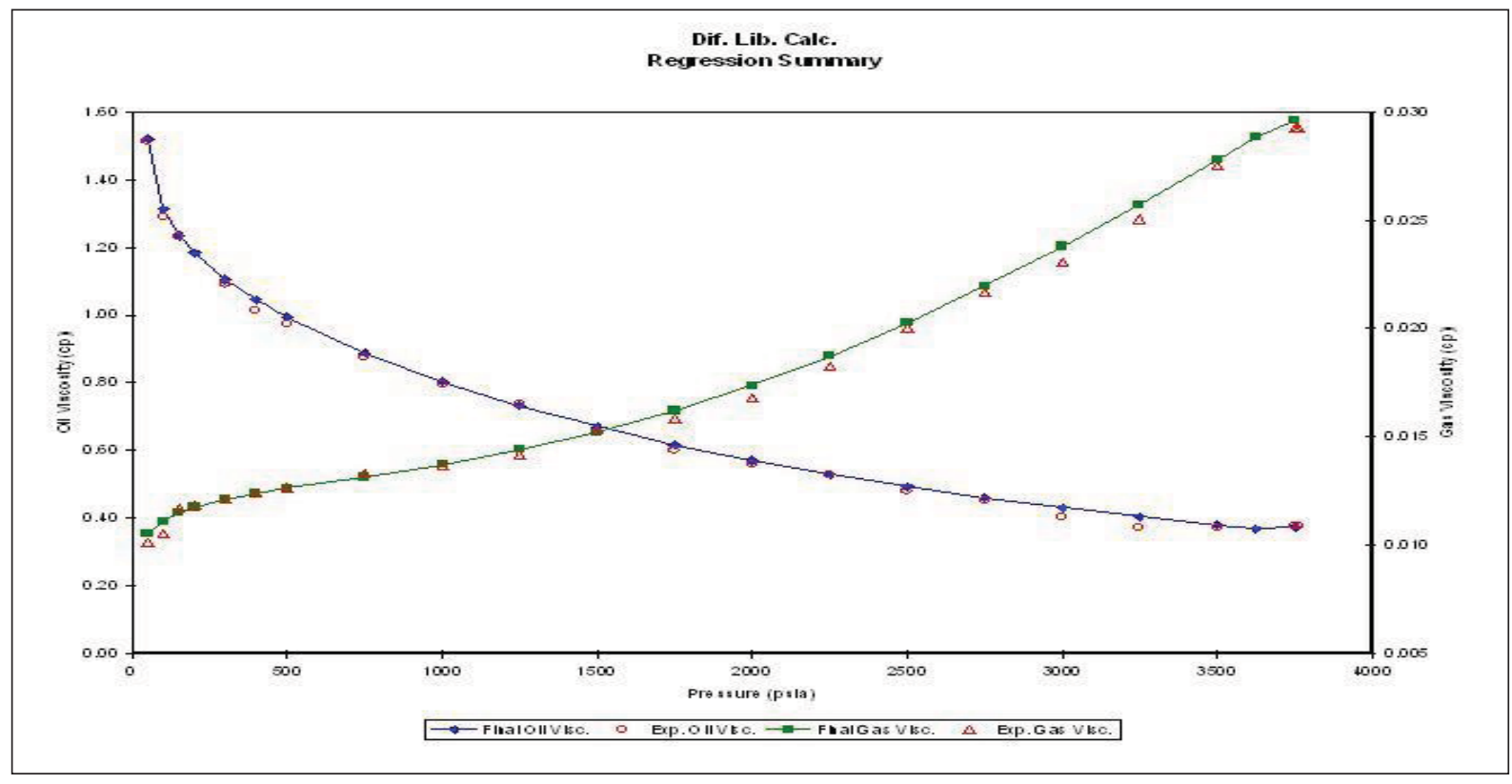

Fig. (4) Oil Viscosity, Gas Viscosity vs. P (SRK method) 


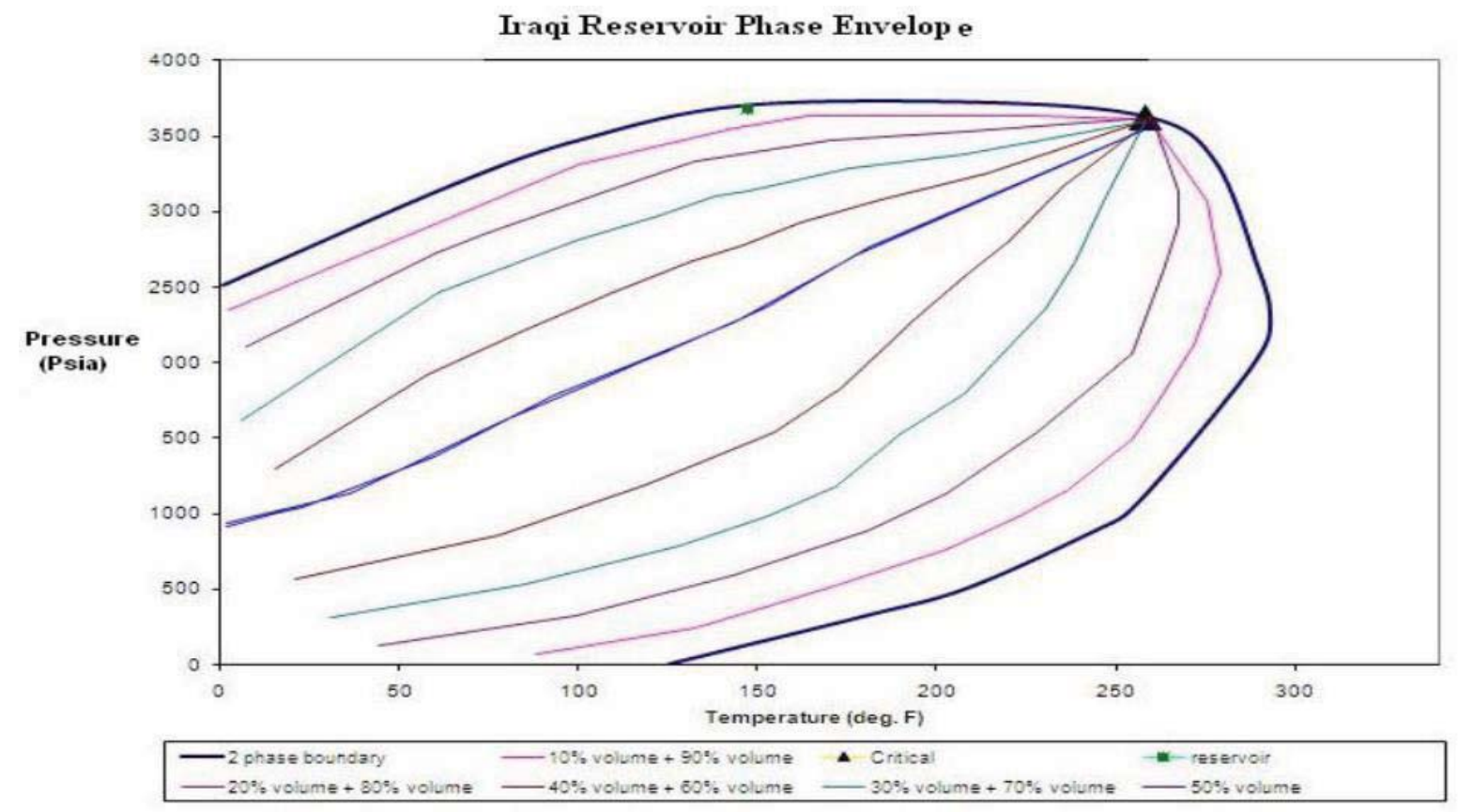

Fig.(5) Phase Envelop of an Iraqi Reservoir

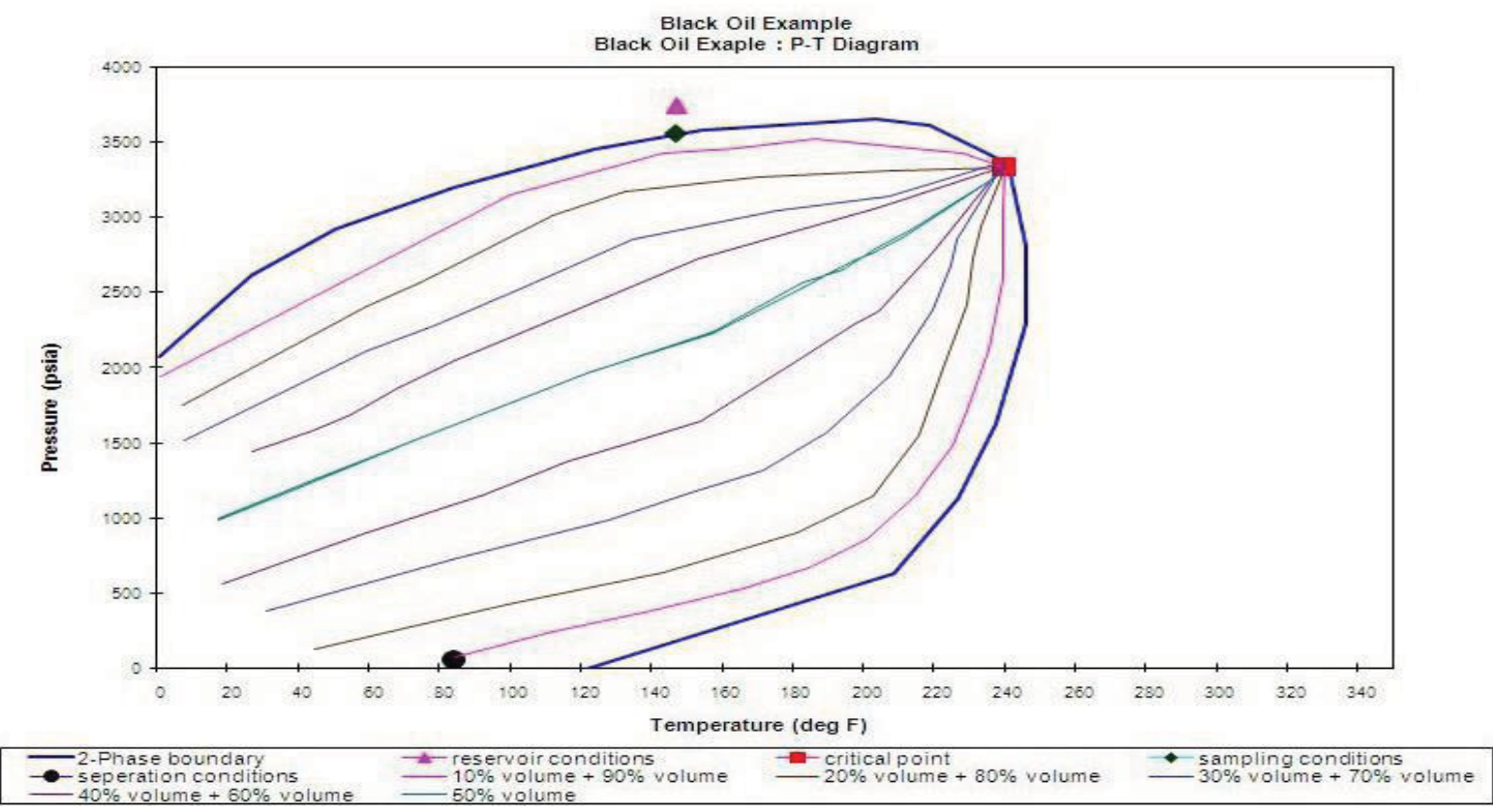

Fig.(6) Oil phase Envelopes 


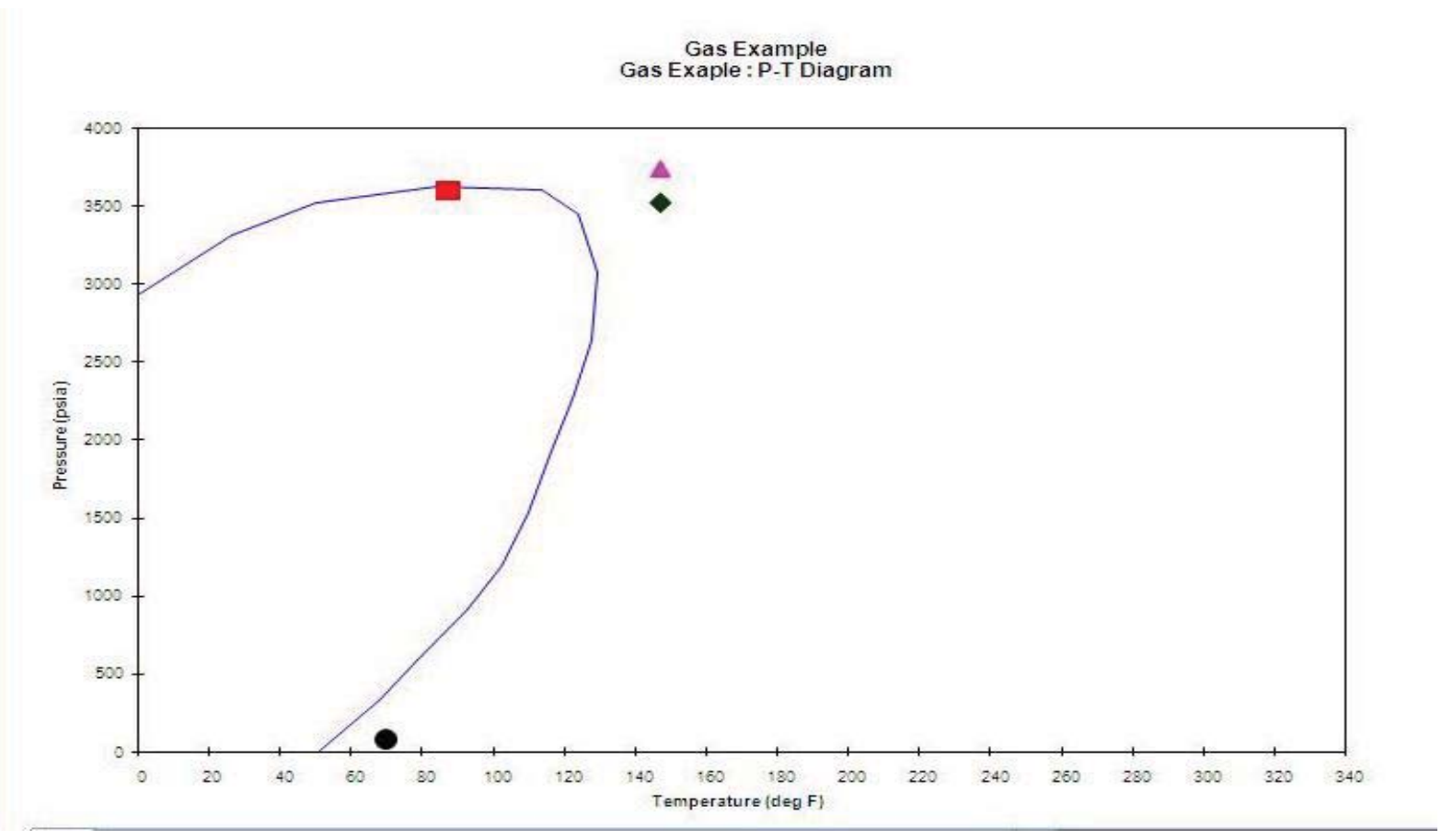

Fig.(7) Gas Cap Phase Envelope for an Iraqi Reservior 


\section{Conclusions}

1-The type of the reservoir fluid was determined from the PVT investigation, as this Iraqi field is a black-oil reservoir with a gas cap, with a high percentage of $\mathrm{C} 1$ hydrocarbons.

2-The SRK was best based on achieving the minimum error percentage comparing its results with the original data

\section{Nomenclature}

B0 = Formation-volume factor, STB/bbl.

$\mathrm{Bg}=\mathrm{Gas}$ formation-volume factor $\mathrm{ft} 3 / \mathrm{Scf}$

GOR $=$ Gas-oil ratio, Scf/STB

$\mathrm{P}=$ Pressure, pisa

$\mathrm{T}=$ Temperature oF

Pc $=$ critical pressure

$\mathrm{Tc}=$ critical temperature

$\mathrm{Vc}=$ critical volume

$\mathrm{Sg}=$ specific gravity

Enth. Coef. $=$ enthalpy coefficient 


\section{No.16 Journal of Petroleum Research \& Studies}

\section{(JPR\&S)}

\section{References}

1. Jihad Husain Al-Joumaa, "A Reservoir Simulation Study of Tertiary Reservoir / Jambour Field”, MSc. Thesis, University of Baghdad, 2011.Petroleum Engineering Department.

2. Robinson, D.B, and Peng, D.Y., "The Characterization of the Heptanes and Heavier Fractions for the GPA Peng-Robinson Programs," Gas Processors Association, Research Report RR-28, March 1978.

3. Soave, G., "Equilibrium Constants from a Modified Redlich-Kwang Equation of State," Chem. Eng. Sci., Vol. 27, 1972, pp. 1197-1203

4. Coats, K.H., Smart, G.T., "Application of a Regression-Based EOS PVT Program to Laboratory Data," SPE Reservoir Eng., Vol. 1, No. 3, May 1986, pp. 277-299

5. Lee, B.I., and Kesler, M.G., "A Generalized Thermodynamic Correlation Based on ThreeParameter Corresponding States," AIChE J., Vol. 21, May 1975, pp. 510-527

6. Twu, C.H., "An Internally Consistent Correlation for Predicting the Critical Properties and Molecular Weights of Petroleum and Coal-Tar Liquids," Fluid Phase Equil., Vol. 16, 1984, pp. $137-150$

7. Riazi, M.R., and Daubert, T.E., "Simplify Property Predictions," Hydrocarbon Processing, March 1980, pp. 115-116

8. Michelsen, M.L., "Calculation of Phase Envelopes and Critical Points for Multi-Component Mixtures," Fluid Phase Equilibrium, Vol. 4, 1980, pp. 1-10 